\title{
Drawing on Paper Maps: Reliable On-line Symbol Recognition of Handwritten Symbols Using a Digital Pen and a Mobile Phone
}

\author{
Tomas Sylverberg $^{1}$, Per Ola Kristensson ${ }^{1,2}$, Ola Leifler ${ }^{1}$, Erik Berglund ${ }^{1,2}$ \\ ${ }^{1}$ Department of Computer and Information Science, Linköping University, Sweden \\ ${ }^{2}$ Santa Anna IT Research Institute, SICS Group, Swedish ICT Institute \\ \{x06tomsy, perkr, olale, eribe\}@ida.liu.se
}

\begin{abstract}
We present a reliable on-line handwritten recognition system which recognizes symbols, such as NATO symbols, drawn on a paper maps using a digital pen and a mobile phone. Our user evaluation shows that maximal accuracy can be achieved. Furthermore, our proposed system has low latency running on offthe-shelf mobile phones. The recognition system is part of a research effort providing field soldiers with lightweight computer support.
\end{abstract}

Keywords: pen-gesture recognition, mobile phones, digital pen, paper-based user interfaces, ubiquitous computing.

\section{Introduction}

There is still extensive use of ordinary paper products within the military while advanced computer systems are relatively unused [8]. The use of computer support is, naturally, desirable given satisfactory cost and mobility characteristics. For the soldier in the field, weight and cost can be imperative design parameters that a computer system for example, a critiquing system, needs to overcome.

The current computerization of physical paper provides us with a new technical platform for advanced computer systems. In this project, as part of the Kartago Research Framework, we explore the development of a paper-map-based computer support tool providing lightweight and low cost characteristics. The Kartago Research Framework focuses on the traditional paper map as the central hardware device and explores the augmentation of paper as a means of providing interactive computer support. Field soldiers use paper maps extensively for, for example, orientation, observation and collaboration. Observations are marked with a military symbol language on the paper map and are also reported to headquarters.

The ability to recognize drawn symbols, such as NATO symbols, is essential in a paper-map-based computer system for soldiers in the field. Symbol recognition is thus a non-trivial area of research. Furthermore, it is challenged by the military domain and by its use in the field.

We present in this paper how the Kartago System funtions in its current form. More precisely, we show how symbol recognition on a printed paper map is can be realized with a digital pen (Anoto) and a mobile phone as main system components. The domain places requirements on the system not addressed by the underlying technology. In particular, the symbol recognition system must be very robust to allow for use in the field as illustrated by Figure 2. Also, the recognition should be handled locally, for instance by the mobile phone.

This system provides pen-based interactivity on a paper map to a computer system running on a mobile phone via wireless communication. Altogether, the hardware configuration assures that the hardware is easily wearable. The system fits the size of a soldier's leg-pocket while retaining all positive aspects of traditional paper maps.

Our user evaluation results show that practically $100 \%$ accuracy $(98 \%$ before training, $100 \%$ after minimal training) is achieved in the Kartago Symbol Recognition running on off-the-shelf mobile phones. Though this initial evaluation was performed under good conditions, we are confident that the symbol recognition part of the Kartago System will provide sufficiently in the field.

\section{Symbol Recognition in the Field}

Armies invest enormous sums in technology to keep up with the technological development. However, in the field, paper still rules. Whereas paper maps do have several advantages over ordinary computerized systems, they do not provide automated connectivity to other military systems.

One important step towards the next generation of soldier equipment is the digitization of soldiers' work. For instance, when a soldier wants to report an observation, this could be done by drawing with a pen on a paper map. One of the challenges with such a system is how to develop, support and sustain the recognition of symbols. 


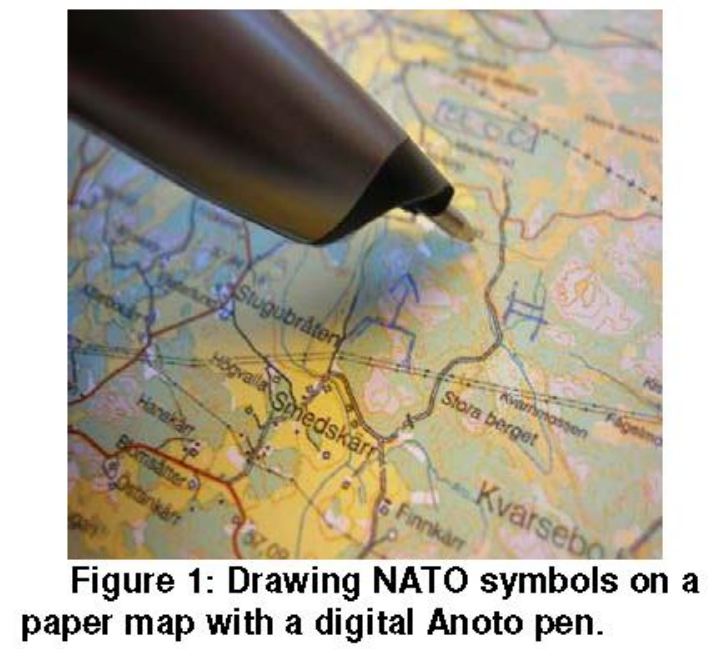

Symbol recognition for military applications requires a domain specific approach. One important aspect is that input data can vary considerably in quality due to the special conditions in which military operations take place. For example, how well a symbol is drawn depends to great extent on what input device is used, if the symbol is drawn by a person who stands or sits, if the canvas is supported by a hard surface underneath or not. This suggests that a solution must be reliable and permissive to bad data.

Although symbol recognition is not a new phenomenon, it unveils the full potential of a mobile military computerized system. Annotations on a map can be captured and used locally, or by remote systems, for instance, by command and control systems.

\section{The Kartago Project}

Kartago is a research project focused on a mobile paper-based or paper-like interactive map platform. Its if focused use in the field for planning, simulation, communication, and documentation. It is an alternative approach to digitization of map-related tasks, which focus on augmenting the traditional paper map as means of digitizing map-related work practice.

Our research project envisions that paper or a paperlike material (such as a flexible screen) can be the central artifact in an interactive-map system where networking, communication and user interactivity can be added on a level as advanced as that of an everyday computer.

The goal is to achieve a printable leg-pocket sized system with a map-sized interface (folded and carried in hand or in a leg-pocket) with symbol-based interaction, and information/communication task management (see Figure 2).

A highly relevant part of the Kartago Platform is symbol management and symbol recognition for the use of hand-written symbols in interactive systems such as critiquing systems. Being able to use the symbol

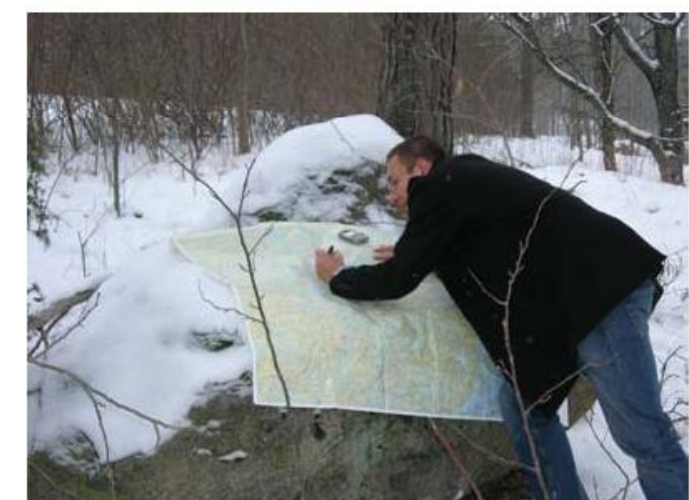

Figure 2: The system in its natural work envir onment.

language already in place is an important part of a mobility platform for the military.

\section{Interactive Maps in the Field}

In a regular computer-enabled staff environment, a multitude of systems can provide decision support for staff members. Mixed-initiative planning systems can help staff officers analyze a course of action with respect to physical limitations, rules of engagement or other known constraints $[1,3,4,10,14]$. Collaboration systems can analyze the interactions between multiple commanders [9] and thereby help them synchronize their actions.

The usefulness of these systems could be extended greatly if they allowed for field officers to access the same information that is available in a regular staff environment through the use of pen and paper.

\section{System Overview}

The outlined solution uses paper maps together with a cellular phone, and an Anoto pen. The pen deduces where the user is drawing by a special pattern which is printed on the particular paper map. This technology allows the soldier to have a full-size paper map which can be folded and put in a pocket. The mobile phone used is a normal bluetooth cellular phone which supports J2ME. Altogether, this hardware configuration assures that the hardware is easily wearable. This paper focuses on the pen-gesture recognition component of a larger system intended for military use.

Figure 3 illustrates the relationship between the paper maps, the digital pen, the cell phone, and an optional server. The user draws symbols with a digital pen on a paper map. The pen is connected wirelessly to a cell phone, which in turn is connected to a server over the mobile network (e.g. GSM, EDGE, 3G, etc.).

Within the framework of the Kartago System for Cellular Phones, the symbol recognition is one 


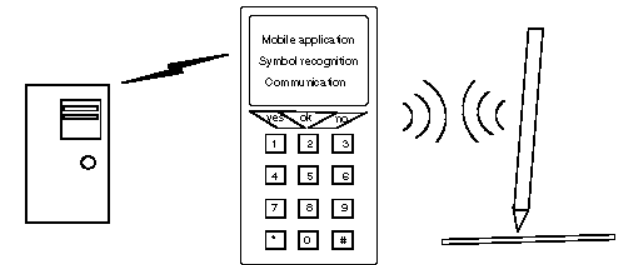

Figure 3: Overview of the pengesture recognition component.

important component. Implemented, the symbol recognition allows the soldier to draw a symbol, and get it recognized. When the symbol is recognized the soldier is allowed to position the symbol and can, optionally, add information which can be used by a critiquing system. The information collected during this work is attached to an XML-representation of the map which can be sent through SMS/MMS, or web services to other soldiers or to headquarters.

\section{Requirements}

The military domain and more specifically the field soldier work practice place particular requirements on symbol recognition and the Kartago System need to be well adapted to these.

\subsection{Support Complex Pen-gestures}

Military symbols are typically complex compound symbols consisting of a combination of various penstrokes (see Figure 4 for examples). Therefore, the pengesture recognizer must be able to handle multiple penstrokes.

\subsection{Reliable}

Since the pen-gesture recognition system is intended to be used in the field, it must be reliable to distortions and maintain high accuracy.

\subsection{Support Low-Powered Devices}

Yet another difficulty is the limited processing power of cellular phones which limits the amount of calculations that can be performed. Also, mobile phones typically lack a floating point unit (FPU).

\subsection{Easy to learn}

The recognition system should work with a minimal amount of training required by the users. In the military domain, the use of predefined symbol languages facilitates the learning process because soldiers can be expected to know the symbols.

\subsection{Easy to maintain}

Many recognition systems are based on training-data where several writing samples for each symbol are stored into a database. A side-effect of the data-driven approach is that it is harder to introduce new symbols since it is not enough to submit one ideal template symbol (e.g. from a handbook of symbols). Instead, several symbols need to be written using a specialized software. As such, a requirement is also that it is easy to maintain the symbol set and add new symbols with minimum user effort.

\section{Recognition}

Cooperation between different national armies creates new challenges to the world of symbol representation. Whereas NATO has one symbol language, separate countries may use distinct systems. In order to support different military symbol languages a special symbol format was developed, which permits the symbol recognition engine to be loaded with different symbol languages. Figure 4 shows a selection of the symbols that the system recognizes.

The symbol format is based on XML, which is a natural choice given that the system, of which the symbol recognizer is one part, is XML-driven. The system provides support to load symbols expressed in our XML-format. A symbol is represented by various attributes, such as an image file, a sound file, and a ordered set of points defining the symbol. The symbol recognizer only requires the ordered set of points, together with information regarding whether the symbol could be rotated, and how far it should be between each rotation of the symbol. The rest of the specifiers are optional and are used by other parts of the application to enhance the user's experience. The implication of requiring an ordered set of points is that drawing order becomes important. It is therefore necessary that the for the user to learn in which order a symbol should be constructed, which as a disadvantage have implications for how easy the system is to learn.

\subsection{Related Work.}

A common pen-gesture recognizer is the Rubine recognizer [13]. The Rubine recognizer is the "classic" statistical linear machine that extracts a feature vector from the input signal and attempts to find the most similar class by statistical inference. The features in the Rubine recognizer are, for example, initial angle, and the length of the stroke [13]. The Rubine recognizer has been integrated into many toolkits, for example SATIN [5]. The Rubine recognizer approach is practical for a few pen-gestures but is increasingly less reliable as the number of symbols increases. For instance, the dependency of a feature such as initial stroke angle 


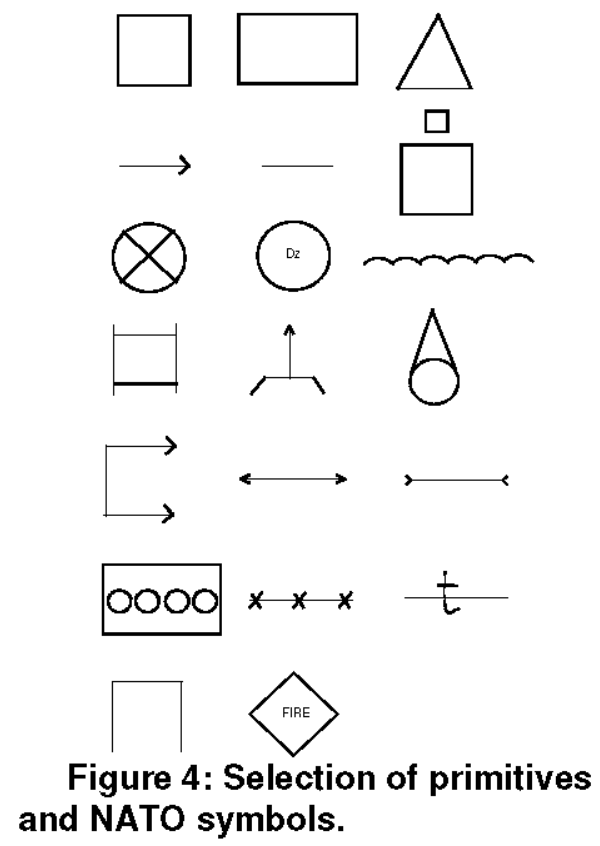

means that if the users initiated an inappropriate angle of attack when starting the pen-gesture it could directly lead to an unintended response. Analytically, another disadvantage with the Rubine recognizer is the possibility to create two geometrically distinct symbols that are completely ambiguous to the Rubine recognizer's feature set.

\subsection{Algorithms.}

The symbol recognition is template-based and the symbol templates are used by the recognizer to identify the user's intended symbol. User input is saved as ordered strokes and is equidistantly sampled and normalized in scale and translation to permit comparison with the reference pattern.

The actual symbol recognition is performed when the unknown symbol has passed the preprocessing steps for recognition. For each template symbol, the distance between the pattern drawn with the digital pen and the reference symbol pattern is calculated by the scoring function $q$ (Equation 1). To improve recognition accuracy we also give extra weight to the start and end point comparisons between the input signal (drawing trace) and the template pattern. In Equation $1 \alpha$ is the weight of the start and end points. In the summation the distance between each ordered pair is summed up:

$q(x, y)=(1-\alpha)\left(\sum_{==1}^{m-1}\left\|x_{i}-y_{i}\right\|\right)+\alpha \gamma(x, y)$

where $x$ and $y$ are two patterns, the subscripted $x_{i}$ and $y_{i}$ components refer to individual sample points and $\gamma$ is a function defined as:

$$
\gamma(x, y)=\left\|x_{0}-y_{0}\right\|_{2}+\left\|x_{\{m-1)}-y_{(m-1)}\right\| .
$$

The recognition engine is based on the concept of proportional matching presented by Kristensson and Zhai [6]. Points are matched on a one-to-one basis as opposed to the elastic matching approach which was used in some early handwriting recognition systems [15]. There are three primary advantages to proportional matching in relation to elastic matching [6]. First, time complexity of the proportional matching algorithm is linear in relation to the number of sample points. By contrast, elastic matching has quadratic time complexity. Second, proportional matching is easier to implement. Third, proportional matching is easier to work with analytically. For instance, to compare elastic matching scores against each other, the scores need to be normalized. However the normalization procedure for elastic matching is non-trivial [7]. Other approaches to recognizing on-line writing (mainly data-driven handwriting recognition) have been proposed [2, 11] (see also Tappert et al. [16] for an extensive review). Since our system is template-based, rather than data driven, new symbols can be added by simply inserting an ideal template of a symbol (e.g. from a handbook) into the recognizer's set of reference symbols. To make proportional matching work well in a military setting, we found it necessary to introduce a couple of modifications of the concept. For military applications in the field, special care has to be taken to how a stroke should be represented. Several systems save strokes as $(x, y, t)$ triplets. In a military setting a symbol recognition that depends on movement dynamics is probably not reliable. A soldier in the field wearing thick gloves will probably not draw as fast as he would do sitting at a desk. Furthermore, expert users have dramatically different movement patterns than novice users or users under considerable stress who may suffer considerable hand tremor. As a result, instead of taking into account when a certain stroke was generated, the order of the strokes is considered. This increases the tolerance toward disruptions in the input procedure. Furthermore, given the risk of unwanted pen-up events the representation of the symbols discards any pen-up and pen-down data. Figure 5 shows the result of how a symbol is represented internally, when pen-up and pendown is discarded.

As has earlier been mentioned, most military symbols are compound symbols, which means that they can be seen as various separate symbols. One approach to recognize compound symbols could be to incrementally recognize each part of the symbol. This could be achieved by on pen-up events triggering a timer which would force the user to continue drawing within a specified amount of time in order to recognize 

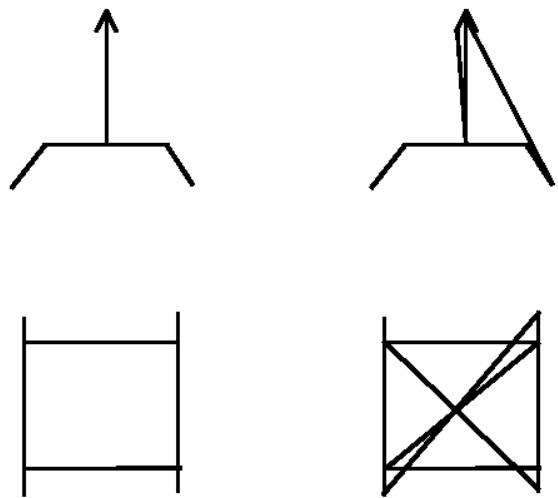

Figure 5: Graphical representation (left) and internal representation (right) of two symbols.

the following strokes as part of the initial symbol. Instead, the

approach in the implemented symbol recognizer looks at the entire symbol when it is drawn and ignores pen-up and pen-down which results in a continuous symbol.

The negative aspect of this approach is that the representation of large and detailed symbols requires a higher sample rate than what would be necessary if the recognizer would use basic primitives to create the symbols. The benefit of the approach is that each symbol has a unique representation, and there is no need to take into consideration where a detected symbol part is located compared to the rest of the symbol's parts. Further, using a set of primitives to create complex symbols would require a greater effort from the user to be able to create new symbols.

As is always the case when developing recognitionbased systems there are multiple conflicting dimensions such as high accuracy, ease-of-use, fast recognition response time. In this application we gave priority to high accuracy (reliable recognition) at the cost of initial ease-of-use.

\section{Empirical Evaluation}

There are many research questions when developing a new recognition system for soldiers in the field. In this paper we have focused on the most fundamental questions: 1. Is recognition reliable enough to be practical? 2. Can the system recognize symbols fast enough to run on off-the-shelf mobile phones?

\subsection{Participants}

Fifteen participants were recruited. They were told to draw the twenty compound pen-gestures depicted in figure 4 . The ages ranged from 27 to 50 (average 40 ). 11 were female and 4 were male. The participants were office workers and therefore had limited or no previous experience with military symbol languages. However, if this was a factor it could be assumed that military personnel would get even better results.

\subsection{Material}

The participants were asked to reproduce 20 pengestures (Figure 4). Each pen-gesture was re-sampled into 100 equidistant sample points.

\subsection{Apparatus}

An Anoto pen model Maxell DP-201 was used as the digital pen. The Anoto pen was used on paper sheets with Anoto's background pattern imprinted. Using the background paper the Anoto pen transmitted absolute and relative positional coordinates to a desktop computer executing the recognizer software. The performance tests were conducted on a Sony Ericsson P990i mobile phone with a $250 \mathrm{MHz}$ 32-bit ARM9 CPU.

\subsection{Procedure}

The experiment was run in two sessions. During the first session, participants were shown how to draw the symbols in Figure 4. Thereafter, they were instructed to reproduce them. The second session was performed after the users had been allowed to practice drawing the symbols that were difficult to, or they had failed to draw, in the first attempt. The participants learned to draw the symbols after having drawn 2-3 times. The experiment was conducted with the participants drawing while sitting at a desk and the results are therefore representative of good field conditions.

\section{Results}

As can be seen in Table 1, the error rate at the first test was well over the acceptable level $(2 \%)$. After minimal training the error rate reduced to zero. The results show that after minimal training very high accuracy is made possible with our proposed symbol recognition system using NATO symbols, and it is clearly practical for prototype testing in the field.

Table 1. Error rates before and after training

\begin{tabular}{|c|c|c|}
\hline & First Test & Second Test \\
\hline Error Rate & $2 \%$ & $0 \%$ \\
\hline
\end{tabular}

Latency was calculated by recognizing five randomly chosen symbols from the symbol set in Figure 4. The average latency was 589 milliseconds. Latency varied from $492 \mathrm{~ms}$ up to $703 \mathrm{~ms}$. The variation can be 
attributed to how complex the input data was to process (re-sample and normalize). We want to emphasize that the latency data points given above are preliminary since they were obtained without using any pruning or indexing mechanisms. We are in the process of developing a filter that prunes out improbable patterns early in the recognition process to reduce latency.

\section{Conclusion}

A symbol recognizer capable of recognizing, for example, hand-drawn NATO symbols is an essential component in a paper-map-based computer system supporting information and communication tasks for field soldiers. This paper shows that the presented Kartago Symbol Recognizer provides a very reliable solution. High accuracy is achievable using the symbol recognizer after minimal training.

The Kartago System illustrates that interactive and mobile computer systems with lightweight and low-cost characteristics can be provided by augmenting papermaps. Combined with technologies included in off-theshelf mobile phones such as GPS, Bluetooth and GPRS/3G, we can support advanced information tasks in the field.

The fact that the system is wearable, while providing the use of full-size maps, suggests that Kartago is a usable tool for military work practice. The work presented here is a step towards real-time interactive computer support in the field.

Against the background of the positive laboratory results of our symbol recognizer, we plan to implement and evaluate the use of the Kartago combined with a plan critiquing system, which would allow us to study the use of interactive paper maps in the field.

\section{Acknowledgements}

This work was in part sponsored by the Swedish National Defence College, Anoto $\mathrm{AB}$ and Santa Anna IT Research Institute AB (SICS Linköping).

\section{References}

[1] Becker, M.A. and Smith, S.F. Mixed-initiative Resource Management: The AMC Barrel Allocator. Proc. AIPS $2000-$ 5th International Conference on Artificial Intelligence Planning and Scheduling.

[2] Bellegarda, E.J., Bellegarda, J.R., Nahamoo, D., and Nathan, K.S. A Fast Statistical Mixture Algorithm for On-line Handwriting Recognition. IEEE Transactions on Pattern Analysis and Machine Intelligence 16, 12, 1227-1233, 1994.

[3] Bresina, J.L., Jónsson, A.K., Morris, P.H., and Rajan, K. Mixed-initiative Planning in MAPGEN: Capabilities and Shortcomings. Proc. IJCAI-2005 Workshop on MixedInitiative Planning and Scheduling.
[4] Fdez-Olivares, J., Castillo, L., García-Pérez, O., and Palao, F.. Bringing Users and Planning Technology Together. Experiences in SIADEX. Proc. ICAPS $2006-16$ th International Conference on Automated Planning and Scheduling.

[5] Hong, J.I. and Landay, J.A. SATIN: A Toolkit for Informal Ink-based Applications. Proc. UIST $2000-13$ th Annual ACM Symposium on User Interface Software and Technology, pp. 63-72.

[6] Kristensson, P.O. and Zhai, S. SHARK ${ }^{2}$ : A Large Vocabulary Shorthand Writing System for Pen-based Computers. Proc. UIST 2004 - 17th Annual ACM Symposium on User Interface Software and Technology

[7] Marzal, A. and Vidal, E. Computation of Normalized Edit Distance and Applications. IEEE Transactions on Pattern Analysis and Machine Intelligence 15, 9, 926-932, 1993.

[8] McGee, D.R., Cohen, P.R., and Wu, L. Something From Nothing: Augmenting a Paper-based Work Practice via Multimodal Interaction. Proc. DARE $2000-A C M$ Conference on Designing Augmented Reality Environments, pp. 71-80.

[9] Myers, K.L., Jarvis, P.A., and Lee, T.. CODA: Coordinating Human Planners. Proc. ECP 2001 - 6th European Conference on Planning.

[10] Myers, K.L., Jarvis, P.A., Tyson, W.M., and Wolverton, M.J. A Mixed-initiative Framework for Robust Plan Sketching. In Proc. ICAPS $2003-13^{\text {th }}$ International Conference on Automated Planning and Scheduling.

[11] Okumur, D., Uchida, S., and Sakoe, H. An HMM Implementation for On-line Handwriting Recognition based on Pen-coordinate Feature and Pen-Direction Feature. Proc. ICDAR 2005 - IEEE International Conference on Document Analysis and Recognition, pp. 26-30.

[12] Perlin, K. Quikwriting: Continuous Stylus-based Text Entry. Proc. UIST 1998 - 11th Annual ACM Symposium on User Interface Software and Technology, pp. 215-216.

[13] Rubine, D. Specifying Gestures by Example. Proc SIGGRAPH 1991 - 18th Annual ACM Conference on Computer Graphics and Interactive Techniques, pp. 329-337.

[14] Smith, S.F., Hildum, D.W., and Crimm, D.R. Comirem: An Intelligent Form for Resource Management. IEEE Intelligent Systems 20, 2, 16-24, 2005.

[15] Tappert, C.C. Cursive Script Recognition by Elastic Matching. IBM Joumal of Research and Development 26,6 , $765-771,1982$.

[16] Tappert, C.C., Suen C.Y. And Wakahara, T. The State of the Art in On-Line Handwriting Recognition. IEEE Transactions on Pattern Analysis and Machine Intelligence $12,8,787-808,1990$. 